\title{
The FIGO Stage IVA Versus IVB of Ovarian Cancer Prognostic Value and Predictive Value for Neoadjuvant Chemotherapy
}

\author{
Parvin Tajik, MD, PhD, $*+$ Roelien van de Vrie, MD, $\neq$ Mohammad H. Zafarmand, MD, PhD, $\neq$ \\ Corneel Coens, MSc, $\S$ Marrije R. Buist, MD, PhD, $\neq$ Ignace Vergote, MD, PhD,// \\ Patrick M.M. Bossuyt, PhD, $\dagger$ and Gemma G. Kenter, MD, PhD
}

\begin{abstract}
Objective: The revised version of the International Federation of Gynaecology and Obstetrics (FIGO) staging system (2014) for epithelial ovarian cancer includes a number of changes. One of these is the division of stage IV into 2 subgroups. Data on the prognostic and predictive significance of this classification are scarce. The effect of neoadjuvant chemotherapy (NACT) versus primary debulking surgery (PDS) in relation to the subclassification of FIGO stage IV is also unknown.

Methods: We used data of the EORTC 55971 trial, in which 670 patients with previous stage IIIC or IV epithelial ovarian cancer were randomly assigned to PDS or NACT; 160 patients had previous stage IV. Information on previous FIGO staging and presence of pleural effusion with positive cytology were used to classify tumors as either stage IVA or IVB. We tested the association between stage IVA/IVB and survival to evaluate the prognostic value and interactions between stage, treatment, and survival to evaluate the predictive performance.

Results: Among the 160 participants with previous stage IV disease, 103 (64\%) were categorized as stage IVA and $57(36 \%)$ as stage IVB tumors. Median overall survival was 24 months in FIGO stage IVA and 31 months in stage IVB patients $(P=0.044)$. Stage IVB patients treated with NACT had 9 months longer median overall survival compared with IVB patients undergoing PDS $(P=0.025)$, whereas in IVA patients, no significant difference was observed (24 vs 26 months, $P=0.48$ ).

Conclusions: The reclassification of FIGO stage IV into stage IVA or IVB was not prognostic as expected. Compared with stage IVA patients, stage IVB patients have a better overall survival and may benefit more from NACT.
\end{abstract}

Key Words: Ovarian cancer, New FIGO classification, Stage IV epithelial ovarian cancer, Overall survival, Subgroup, Lymph node metastasis

Received June 30, 2017, and in revised form September 28, 2017.

Accepted for publication September 28, 2017.

(Int J Gynecol Cancer 2018;00: 00-00)

Departments of *Pathology and $†$ Clinical Epidemiology, Biostatistics, and Bioinformatics, Academic Medical Centre; $\ddagger$ Department of Obstetrics and Gynaecology, Centre for Gynaecologic Oncology Amsterdam, Academic Medical Centre, Amsterdam, the Netherlands; §The European Organization for Research and Treatment of Cancer Headquarters, Brussels, Belgium; and \|Department of Obstetrics and Gynaecology, Division of Gynaecological Oncology and Leuven Cancer Institute, University Hospitals, K. U. Leuven, Leuven, Belgium.

Address correspondence and reprint requests to Parvin Tajik, $\mathrm{MD}, \mathrm{PhD}$,

Department of Pathology, Academic Medical Centre - University of

Copyright (C) 2018 by IGCS and ESGO

ISSN: $1048-891 \mathrm{X}$

DOI: $10.1097 /$ IGC.0000000000001186
Amsterdam, Meibergdreef 9, 1100 DD, Amsterdam, the Netherlands Room M2-106; PO Box 22660. E-mail: P.Tajik@amc.uva.nl.

Parvin Tajik was supported by an AXA Research Fund. The EORTC 55971 trial was supported by grants (2U10 CA11488-28 through 2U10 CA011488-36) from the National Cancer Institute and by a donation from Vlaamse Liga Tegen Kanker (the Flemish League Against Cancer) to the EORTC Charitable Trust.

The authors declare no conflicts of interest.

PT and RVV contributed equally to this work.

Trial registration: The EORTC-55971 study is registered with ClinicalTrials.gov, number NCT00003636.

Supplemental digital content is available for this article. Direct URL citation appears in the printed text and is provided in the HTML and PDF versions of this article on the journal's Web site (www.ijgc.net). 
C lassification of malignancies is intended to predict the prognosis of patients at different stages of disease. For ovarian cancer, the International Federation of Gynaecology and Obstetrics (FIGO) staging is the most widely used classification system. The first FIGO classification on ovarian cancer was presented in $1976^{1}$; it was updated in $1988^{2}$ and in $2014 .^{3}$ Higher FIGO stages correspond with a poorer prognosis. ${ }^{4}$

One of the adjustments of the new FIGO stage classification of 2014 was the modification of FIGO stage IV by introducing stage IVA and IVB. ${ }^{3}$ The FIGO stage IV disease had been defined as tumor spread outside the abdominal cavity, including malignant pleural effusion, and/or visceral metastases. The new FIGO stage IVA incorporates patients with pleural effusion with positive cytology. The FIGO IVB incorporates patients with parenchymal metastases (eg, liver, spleen, and lung) and metastases to extraabdominal organs. The incidence of FIGO stage IV ovarian cancer seems to have risen over the past decades from about $13 \%$ to $30 \%$, which could be due to more intensive preoperative workup and more comprehensive staging with application of serum tumor markers and advanced imaging modalities as well as laparoscopy and thoracoscopy. ${ }^{5,6}$ Sometimes, upstaging of stage IIB to IIIC to the new FIGO stage IVB might occur based on transmural bowel infiltration. However, this event seems to be rare $(5 \%$ reported in the literature). ${ }^{7}$

Few studies have investigated the prognostic value of the new subclassification. Toptas et $\mathrm{al}^{8}$ observed a better survival for IVA patients, whereas Rosendahl et $\mathrm{al}^{9}$ showed a similar overall survival for IVA or IVB patients. ${ }^{9}$ In contrast, Paik et al ${ }^{10}$ and Ataseven et $\mathrm{al}^{7}$ found a better survival for stage IVB compared with IVA. ${ }^{7,11}$ None of these studies found a statistically significant difference.

We previously investigated the prognostic and predictive value of previous FIGO IIIC and IV staging in a randomized trial and found clinical stage to be prognostic as well as predictive. Staging was associated with the extent of benefit from primary debulking surgery (PDS) compared with neoadjuvant chemotherapy (NACT). ${ }^{12}$

The aim of the analysis presented here was twofold. We wanted to investigate whether the new FIGO IV classification, with a separation into stage IVA and stage IVB, added prognostic information for stage IV patients. We also evaluated whether the classification to IVA and IVB was associated with a benefit from PDS relative to NACT. If so, the subclassification could guide clinical decision-making. For these analyses, we used data collected in the EORTC-55971 study, a randomized trial comparing PDS with NACT. ${ }^{13}$

\section{PATIENTS AND METHODS}

\section{Study Design and Patients}

The background, design, and conduct of the EORTC55971 trial (ClinicalTrials.gov, number NCT00003636) have previously been reported in detail elsewhere. ${ }^{13}$ The trial was conducted in 59 centers in 5 European countries, United Kingdom, and Canada. The study was approved by the EORTC Protocol Review Committee and the NCIC Clinical Trials Group Clinical Trials Committee. Before receiving treatment, all patients provided written informed consent. Eligible to participate were women with cytology or biopsy-proven stage IIIC or IV epithelial ovarian, fallopian-tube, or primary peritoneal cancer.

Between September 1998 and December 2006, eligible and consenting women were randomly allocated to either PDS followed by platinum-based chemotherapy or to platinumbased NACT followed by interval debulking surgery.

The primary outcome was overall survival measured from the date of randomization to the date of death, whatever the cause. Secondary outcomes were adverse effects and progression-free survival. Follow-up was measured for 5 years.

\section{Data Analysis}

The design and conduct of the EORTC 55971 trial was before the introduction of the new FIGO stage classification. Therefore, the analyses of this study were not planned in the original trial protocol.

For this analysis, we selected patients with stage IV disease by the old FIGO classification system and recategorized them with respect to the new FIGO classification ${ }^{3,5}$ using information recorded at baseline. In the new FIGO classification, patients with only malignant pleural effusion or pleural lesions were categorized into stage IVA. Patients with parenchymal metastases and metastases to extraabdominal organs (including inguinal lymph nodes and lymph nodes outside of the abdominal cavity) with or without pleural effusion or pleural lesions were categorized into stage IVB.

Differences in patient characteristics between stage groups were assessed for significance by $\chi^{2}$ test statistics. Binary logistic regression was conducted to assess the association between stage and debulking status, adjusting for the allocated treatment. Univariable and multivariable survival analyses were performed using Kaplan-Meier and Cox proportional hazards models, respectively. To evaluate the prognostic performance, we tested the association between FIGO stage and survival. To evaluate the predictive performance, we tested for interaction between FIGO stage, treatment (PDS or NACT), and survival. All analyses were done based on intention-to-treat principle. We used R for Windows (version 3.2.5; R Foundation for Statistical Computing, Vienna, Austria).

\section{RESULTS}

\section{Patients' Characteristics}

Of the 670 trial participants, $160(24 \%)$ had FIGO stage IV disease. The remaining 510 patients had FIGO stage IIIC disease and were excluded from further analyses. Patient characteristics of stage IV patients are summarized in Supplementary Table S1, http://links.lww.com/IGJ/AS89.

Of the stage IV patients, 79 (49\%) had been randomly allocated to PDS and $81(51 \%)$ to NACT. Debulking to no residual disease was achieved in 51 patients (37\%): $20(30 \%)$ in the PDS group and $31(44 \%)$ in the NACT group.

The FIGO stage IV was diagnosed based on the presence of pleural effusion with positive cytology or pleural lesions in 103 patients (64\%; FIGO stage IVA). The remaining 57 patients $(36 \%$; FIGO stage IVB) had 1 or more metastases in extraabdominal organs or liver or spleen. There were no significant differences in baseline characteristics between stage IVA and stage IVB patients except for significantly higher 
levels of serum CA125 in the stage IVA group. The location of the metastases in all stage IV patients is presented in Supplementary Table S2, http://links.lww.com/IGJ/AS90.

\section{Treatment Allocation and Debulking Status}

A higher proportion of stage IVB patients were randomly assigned to PDS (61\%) compared with $43 \%$ of stage IVA patients (Supplementary Table S1, http://links.lww.com/ IGJ/AS89). We observed that stage IVB patients experienced significantly more residual tumor after debulking surgery ( $>1 \mathrm{~cm}$ residue; $41 \%$ vs $27 \% ; P=0.026$, Supplementary Table S1, http://inks.lww.com/IGJ/AS89). In the NACT group, stage was not significantly associated with successful surgery (leaving no macroscopic residual disease) (odds ratio [OR], 1.3; $95 \%$ confidence interval [CI], 0.4-4.0;P=0.6), whereas in the PDS group, stage IVB patients had a significantly higher risk of residual tumor after surgery compared with stage IVA patients (OR, 4.0; 95\% CI, 1.2-13.7; $P=0.028$ ).

\section{Overall Survival}

The last follow-up information of the participants was collected in 2012; 138 women with stage IV were followed until death $(86 \%)$. The median follow-up time among survivors was 55 months (interquartile range, 47-67). The median overall survival (OS) in all stage IV patients was 26 months ( $95 \%$ CI, 21-32).

Overall survival was not associated with allocated treatment $(P=0.117)$. Univariable association of potential prognostic factors of OS is summarized in Table 1. Among the studied factors, FIGO stage IVA/IVB was the only one significantly associated with OS $(P=0.044$; Fig. 1$)$, with better survival for stage IVB.

The median OS in stage IVB patients allocated to NACT was 9 months longer than in IVB patients undergoing PDS (35 vs 26 months, $P=0.025$, Fig. 2), whereas in IVA patients, no significant difference was observed (24 vs 26 months, $P=0.48)$. A statistical test for stage-by-treatment interaction did not reach significance $(P$ for interaction $=0.09)$.

TABLE 1. Univariate analysis of potential determinants of OS in patients with FIGO stage IV

\begin{tabular}{|c|c|c|c|c|}
\hline Risk Factors & Total $(n)$ & Death $(n)$ & Median OS (95\% CI) & $P$ \\
\hline \multicolumn{5}{|l|}{ Age (y) } \\
\hline$\leq 65$ & 106 & 93 & $28(25-34)$ & \\
\hline$>65$ & 54 & 45 & $21(16-33)$ & 0.703 \\
\hline \multicolumn{5}{|l|}{ WHO performance status } \\
\hline 0 & 64 & 52 & $27(25-41)$ & \\
\hline 1 & 70 & 63 & $27(22-34)$ & \\
\hline 2 & 26 & 23 & $20(9-35)$ & 0.195 \\
\hline \multicolumn{5}{|l|}{ Primary site } \\
\hline Ovarian & 139 & 119 & $27(24-33)$ & \\
\hline Other & 21 & 19 & $17(11-46)$ & 0.482 \\
\hline \multicolumn{5}{|l|}{ Histology } \\
\hline HGSOC & 76 & 67 & $24(20-31)$ & \\
\hline Other/unknown & 75 & 64 & $31(25-40)$ & 0.397 \\
\hline \multicolumn{5}{|l|}{ CA125 } \\
\hline$\leq 500$ & 28 & 23 & $23(15-52)$ & \\
\hline$>500$ & 131 & 115 & $26(23-32)$ & 0.787 \\
\hline \multicolumn{5}{|l|}{ Largest metastatic tumor } \\
\hline$\leq 5 \mathrm{~cm}$ & 60 & 50 & $32(26-45)$ & \\
\hline$>5 \mathrm{~cm}$ & 99 & 87 & $24(22-29)$ & 0.071 \\
\hline \multicolumn{5}{|l|}{ FIGO stage } \\
\hline IVA & 103 & 93 & $24(21-32)$ & \\
\hline IVB & 57 & 45 & $31(24-40)$ & 0.044 \\
\hline \multicolumn{5}{|l|}{ Allocated treatment } \\
\hline Primary surgery & 79 & 72 & $26(21-34)$ & \\
\hline Primary chemotherapy & 81 & 66 & $26(23-35)$ & 0.117 \\
\hline \multicolumn{5}{|l|}{ Residual tumor* } \\
\hline No residue $(0 \mathrm{~cm})$ & 51 & 43 & $34(26-44)$ & \\
\hline Any residue $(>0 \mathrm{~cm})$ & 87 & 76 & $26(21-31)$ & 0.080 \\
\hline
\end{tabular}



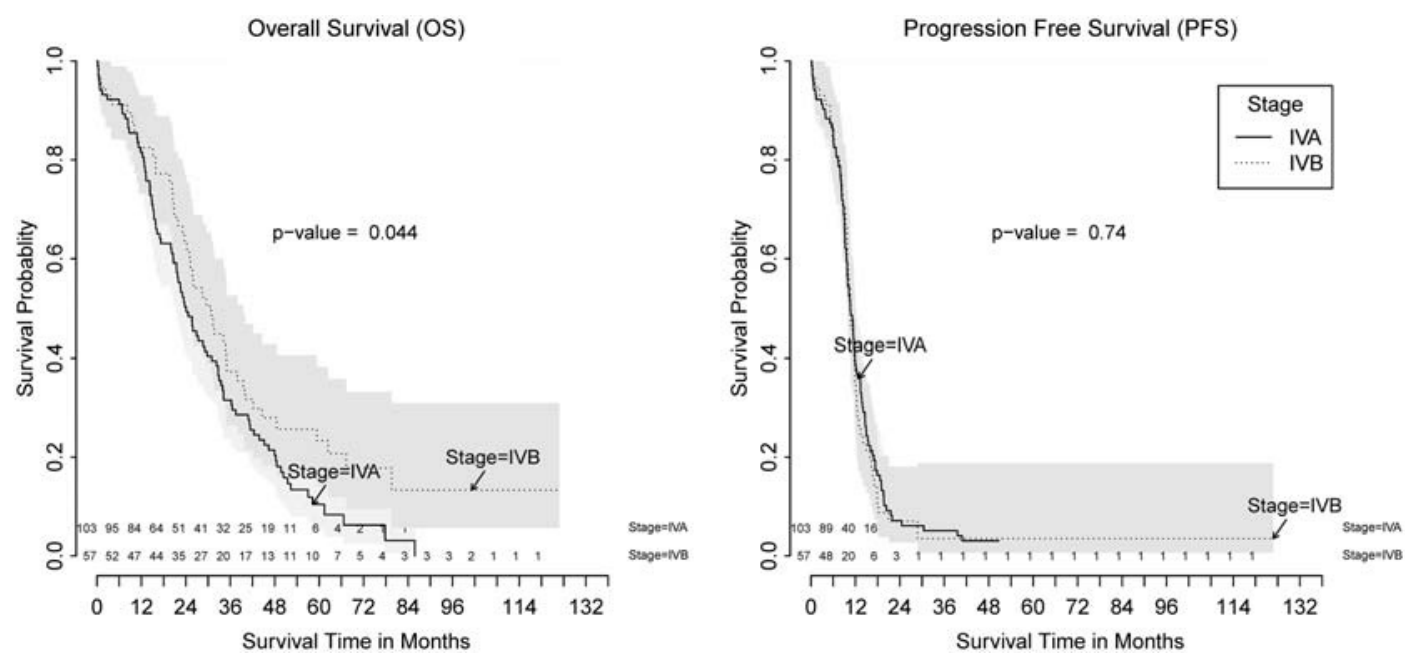

FIGURE 1. Overall survival and PFS in patients with stage IV epithelial ovarian cancer according to subgroup FIGO IVA and FIGO IVB.

In multivariable analysis, including other prognostic factors (Table 2), stage IVB was still associated with a better OS (hazard ratio $=0.45 ; 95 \% \mathrm{CI}, 0.2-0.8 ; P=0.008$ ).

\section{Secondary Outcomes Progression-Free Survival and Perioperative and} Postoperative grade 3 or $\mathbf{4}$ Adverse Events

Recurrence was observed in 153 patients $(95.6 \%)$. The median progression-free survival (PFS) in all stage IV patients was 11 months $(95 \% \mathrm{CI}, 10-12)$. The PFS was not associated with allocated treatment $(P=0.480)$ and did not differ significantly between stage IVA and IVB patients $(P=$ 0.7 ). Patients with no residual disease after debulking surgery had significantly longer PFS than patients with less than $1 \mathrm{~cm}$ or greater than $1 \mathrm{~cm}$ residual tumor after debulking surgery in all FIGO IV patients (12 vs 11 months, $P=0.004)$. No prognostic factors were associated with PFS (Supplementary Table S3, http://links.lww.com/IGJ/AS91).

Grade 3 or 4 adverse events occurred in 49 patients (31\%). Primary debulking surgery was associated with a significantly higher risk of adverse events compared with NACT $(\mathrm{OR}, 3.3 ; 95 \% \mathrm{CI}, 1.6-6.8 ; P=0.001)$. The association between treatment and adverse events was not different between stage IVA and stage IVB patients ( $P$ for interaction 0.95 ).

\section{Prognostic Impact of Metastasis Limited to Extraabdominal Lymph Nodes}

To investigate whether the better survival of the stage IVB group could be partially explained by a better prognosis of a subgroup of stage IVB patients who had only extraabdominal lymph node involvement (including supraclavicular, inguinal, axillary, or mediastinal), we divided the stage IVB group into those with only lymph node metastasis $(n=15)$ and those with
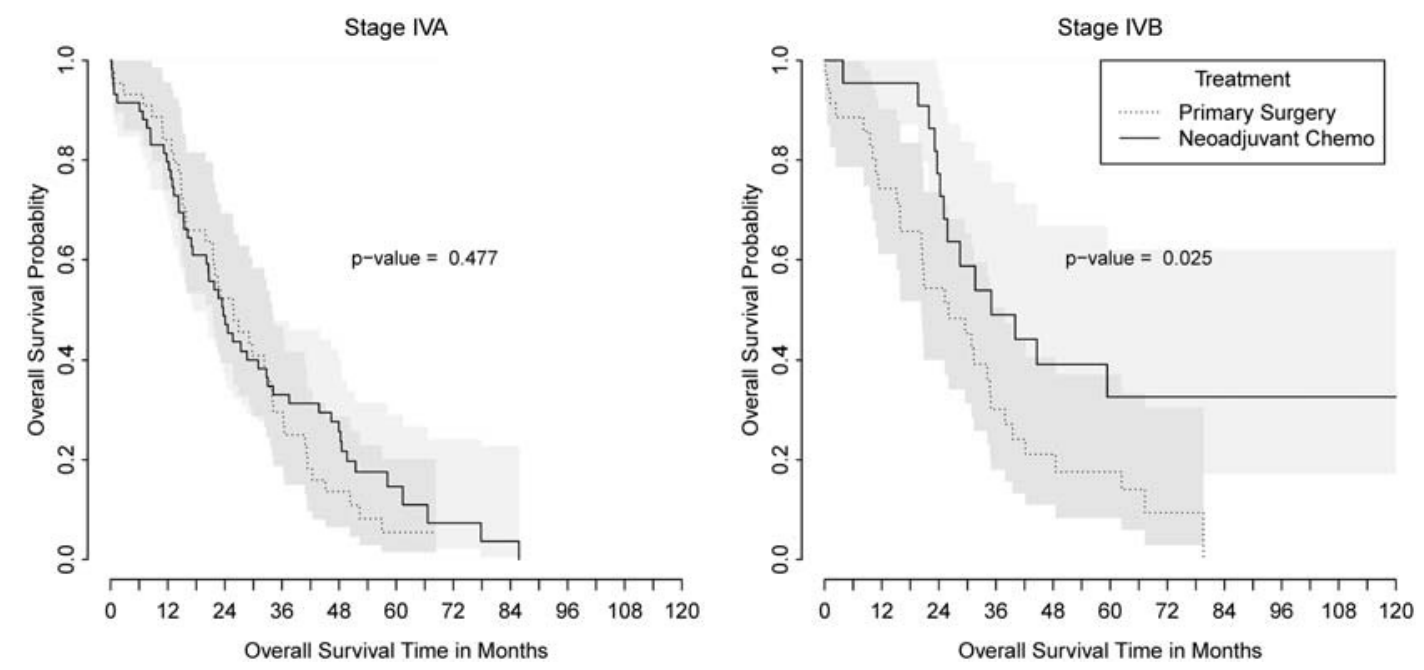

FIGURE 2. Overall survival after PDS or NACT separately for patients with FIGO stage IVA or IVB epithelial ovarian cancer. 
TABLE 2. Multivariate analysis for OS in patients with FIGO stage IV

\begin{tabular}{lccc}
\hline \multicolumn{1}{c}{ Risk Factors } & $\begin{array}{c}\text { Hazard } \\
\text { Ratio }\end{array}$ & $\begin{array}{c}\mathbf{9 5 \%} \\
\text { CI }\end{array}$ & $\boldsymbol{P}$ \\
\hline Age $(\mathrm{y})$ & & & \\
$\quad \leq 65$ & 1 & & \\
$\quad>65$ & 1.11 & $0.8-1.6$ & 0.581 \\
WHO performance status & & & \\
$\quad 0$ & 1 & & \\
1 & 1.37 & $0.8-1.7$ & 0.503 \\
2 & 1.57 & $0.9-2.6$ & 0.084 \\
CA125 & & & \\
$\quad \leq 500$ & 1 & & \\
$\quad>500$ & 0.92 & $0.6-1.5$ & 0.734 \\
Largest metastatic tumor & & & \\
$\quad \leq 5 \mathrm{~cm}$ & 1 & & \\
$\quad>5 \mathrm{~cm}$ & 1.37 & $0.9-1.9$ & 0.111 \\
FIGO stage & & & \\
$\quad$ IVA & 1 & & \\
$\quad$ IVB & 0.43 & $0.2-0.8$ & 0.008 \\
Allocated treatment & & & \\
$\quad$ PDS & 1 & & \\
$\quad$ NACT & 1.12 & $0.7-1.7$ & 0.589 \\
Stage-by-treatment interaction* & 2.08 & $0.9-4.6$ & 0.066 \\
\hline *Stage $\times$ treatment interaction term in the model, which tests if \\
treatment effect is different between stage IVA and IVB subgroups. \\
\hline
\end{tabular}

other organs involved with or without lymph node involvement $(n=42)$. Patients with only lymph node metastasis had significantly better OS than other stage IVB patients $(P=0.038)$. The OS of the rest of the stage IVB patients was similar to stage IVA patients (Fig. 3).

\section{DISCUSSION}

The new FIGO classification adds prognostic information with the further classification of stage IV patients into Stage IVA or IVB ovarian cancer. The difference may go against expectations; we observed a worse OS for patients with stage IVA compared with stage IVB. A positive treatment effect was seen for patients with stage IVB treated with NACT, resulting in better survival compared with treatment with PDS. This might be related to the higher incidence of greater-than-1 $\mathrm{cm}$ residual tumor after debulking surgery after PDS in stage IVB patients.

The main strength of our evaluation of the new FIGO stage IV subclassification is that it is based on data from a randomized controlled trial and, as a result, administration of PDS or NACT was independent of the FIGO stage of the participants or other baseline characteristics. This allows evaluation of the prognostic effect of tumor stage separate from the effect of the administered treatment. So far, the few studies that have investigated the prognostic effect of the new staging have all been retrospective observational studies. Rosendahl et $\mathrm{al}^{9}$ and Paik et al ${ }^{10}$ included patients irrespective of the treatment strategy without further adjustment for the potential treatment effect. Ataseven et $\mathrm{al}^{7}$ and Toptas et $\mathrm{al}^{8}$ restricted their study to patients treated with PDS.

Another strength of our study is the long follow-up time as well as a relatively low rate of censoring (14\% for OS). This provided us with enough events for the associations we studied and increased the statistical power in face of small number of patients.

A limitation, however, is that we only focused on subclassification of former FIGO stage IV patients into IVA and IVB subgroups. We did not account for upstaging of previously FIGO IIB and IIIC patients with exclusively transmural bowel infiltration because the required information was not recorded in the original trial. However, Ataseven et $\mathrm{al}^{7}$ have prospectively analyzed the prevalence of patients with solely transmural bowel infiltration in patients as reason to be classified as FIGO IVB and found this event to be rare (5\%).

Several factors may have contributed to the difference in prognosis between stage IVA and stage IVB patients. There is ambiguity around classification of patients with pleural metastases. In our current analysis, we categorized these patients $(n=2)$ as stage IVA. ${ }^{5,7}$ However, many experts believe that they may have a poorer prognosis, probably similar to that of patients with lung metastases.

Stage IVB is a relatively heterogeneous group, with a mixture of subgroups with possibly good and bad prognosis. The extent of disease can vary from exclusively abdominal wall metastases or only extraabdominal lymph node metastases (limited disease) to extensive tumor growth in various organs or peritoneal carcinomatosis. ${ }^{14,15}$ This may have resulted in an average better prognosis of the IVB group in our study. Some studies have reported better prognosis for advanced ovarian cancers with lymph node involvements alone. ${ }^{16-19}$ This could be one of the reasons why stage IVB patients in our study had a

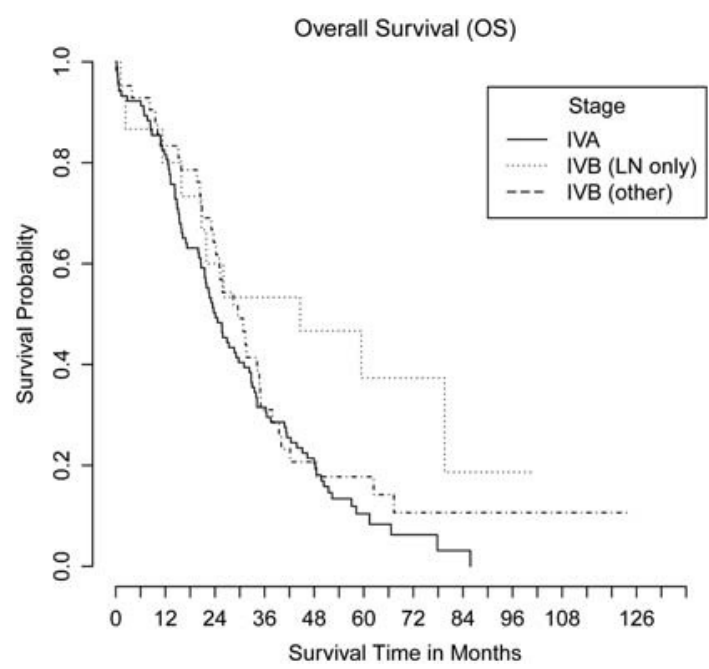

FIGURE 3. Overall survival of patients with stage IVA, stage IVB limited to extraabdominal lymph node metastasis (IVB [LN only]), and stage IVB with organs involved other than lymph nodes (IVB [other]). 
better OS. Our analysis also supports this hypothesis because the 15 patients with only extraabdominal lymph node metastases had significantly better survival than other stage IV patients. Based on this analysis, extraabdominal lymph node involvement, compared with malignant pleural effusion, might better qualify as the decisive factor to subclassify stage IV patients. Because this hypothesis is only based on 15 patients, it needs to be evaluated in a different and larger study group cohort first.

The process of updating the FIGO staging for ovarian cancer was explained by Mutch et al. ${ }^{20}$ The new staging was based on the best available evidence, and consensus was reached by all participants in the FIGO meeting held in Rome, Italy, on October 7, 2012. The committee acknowledged that there are areas that are not supported by strong evidence and has been careful to ensure that changes are not made without quality evidence when available. Extensive research was presented to support the changes in FIGO stage III. In contrast, the changes in FIGO stage IV were not explained or substantiated in this explanatory article.

In conclusion, the reclassification of FIGO stage IV into stage IVA or IVB adds prognostic information and may add predictive information concerning the effect of NACT. Compared with stage IVA patients, stage IVB patients had a better OS and benefitted more from NACT. Whether changing the subclassification of stage IV disease based on lymph node involvement instead of malignant pleural effusion could improve the prognostic and predictive classification of patients is a question requiring further investigation.

\section{REFERENCES}

1. Kottmeier HL. Presentation of therapeutic results in carcinoma of the female pelvis: experience of the annual report on the results of treatment in carcinoma of the uterus, vagina, and ovary. Gynecol Oncol. 1976;4:13-19.

2. Shepherd JH. Revised FIGO staging for gynaecological cancer. Br J Obstet Gynaecol. 1989;96:889-892.

3. Prat J. Oncology FCoG. Staging classification for cancer of the ovary, fallopian tube, and peritoneum. Int J Gynaecol Obstet. 2014;124:1-5.

4. Heintz AP, Odicino F, Maisonneuve P, et al. Carcinoma of the ovary. FIGO 26th Annual Report on the Results of Treatment in Gynecological Cancer. Int J Gynaecol Obstet. 2006;95:S161-S192.

5. Ataseven B, Chiva LM, Harter P, et al. FIGO stage IV epithelial ovarian, fallopian tube and peritoneal cancer revisited. Gynecol Oncol. 2016;142:597-607.

6. Prader S, Harter P, Grimm C, et al. Surgical management of cardiophrenic lymph nodes in patients with advanced ovarian cancer. Gynecol Oncol. 2016;141:271-275.
7. Ataseven B, Harter P, Grimm C, et al. The revised 2014 FIGO staging system for epithelial ovarian cancer: is a subclassification into FIGO stage IVA and IVB justified? Gynecol Oncol. 2016;142:243-247.

8. Toptas T, Pestereli E, Erol O, et al. Validation of revised FIGO staging classification for cancer of the ovary, fallopian tube, and peritoneum based on a single histological type. Int J Gynecol Cancer. 2016;26:1012-1019.

9. Rosendahl M, Hogdall CK, Mosgaard BJ. Restaging and survival analysis of 4036 ovarian cancer patients according to the 2013 FIGO classification for ovarian, fallopian tube, and primary peritoneal cancer. Int J Gynecol Cancer. 2016;26:680-687.

10. Paik ES, Lee YY, Lee EJ, et al. Survival analysis of revised 2013 FIGO staging classification of epithelial ovarian cancer and comparison with previous FIGO staging classification. Obstet Gynecol Sci. 2015;58:124-134.

11. Paik ES, Lee Y-Y, Lee E-J, et al. Survival analysis of revised 2013 FIGO staging classification of epithelial ovarian cancer and comparison with previous FIGO staging classification. Int $J$ Gynecol Cancer. 2015;58:124-134.

12. van Meurs HS, Tajik P, Hof MH, et al. Which patients benefit most from primary surgery or neoadjuvant chemotherapy in stage IIIC or IV ovarian cancer? An exploratory analysis of the European Organisation for Research and Treatment of Cancer 55971 randomised trial. Eur J Cancer. 2013;49:3191-3201.

13. Vergote I, Tropé CG, Amant F, et al. Neoadjuvant chemotherapy or primary surgery in stage IIIC or IV Ovarian Cancer. $N$ Engl $J$ Med. 2010;363:943-953.

14. Ataseven B, du Bois A, Harter P, et al. Impact of abdominal wall metastases on prognosis in epithelial ovarian cancer. Int $J$ Gynecol Cancer. 2016;26:1594-1600.

15. Ataseven B, Grimm C, Harter P, et al. Prognostic impact of debulking surgery and residual tumor in patients with epithelial ovarian cancer FIGO stage IV. Gynecol Oncol. 2016;140:215-220.

16. Suh DH, Kim TH, Kim J-W, et al. Improvements to the FIGO staging for ovarian cancer: reconsideration of lymphatic spread and intraoperative tumor rupture. J Gynecol Oncol. 2013;24: 352-358.

17. Berek JS. Lymph node-positive stage IIIC ovarian cancer: a separate entity? Int J Gynecol Cancer. 2009;19:S18-S20.

18. Cliby WA, Aletti GD, Wilson TO, et al. Is it justified to classify patients to Stage IIIC epithelial ovarian cancer based on nodal involvement only? Gynecol Oncol. 2006;103:797-801.

19. Baek SJ, Park JY, Kim DY, et al. Stage IIIC epithelial ovarian cancer classified solely by lymph node metastasis has a more favorable prognosis than other types of stage IIIC epithelial ovarian cancer. J Gynecol Oncol. 2008;19:223-228.

20. Mutch DG, Prat J. 2014 FIGO staging for ovarian, fallopian tube and peritoneal cancer. Gynecol Oncol. 2014;133:401-404. 